\title{
DESEMPENHO AGRONÔMICO DO RABANETE SOB DIFERENTES COBERTURAS VEGETAIS DO SOLO
}

\author{
GASTL FILHO, Josef ${ }^{1}$ \\ RESENDE, Millena Almeida ${ }^{2}$ \\ FERREIRA, Ismael ${ }^{3}$ \\ LACERDA, Wesley Candido de ${ }^{3}$ \\ SILVA, Marcelo Belisário da ${ }^{3}$ \\ SILVA, Vanessa Aparecida ${ }^{3}$ \\ BARBOSA, Marcelo da Silva ${ }^{3}$
}

\begin{abstract}
Recebido em: 2020.04 .28
Aprovado em: 2020.06 .25

ISSUE DOI: $10.3738 / 1982.2278 .3789$

RESUMO: O aproveitamento na agricultura de resíduos orgânicos oriundos das mais variadas áreas da cadeia produtiva de alimentos é de extrema importância, tendo em vista o seu potencial poluidor e como fonte de nutrientes para o solo. Neste sentido, o presente estudo teve por objetivo avaliar a influência de resíduos orgânicos como cobertura morta vegetal sobre o desempenho agronômico da cultura do rabanete (Raphanus sativus. L.). O experimento foi conduzido em delineamento em blocos casualizado (DBC), com 4 tratamentos (bagaço de cana-deaçúcar; casca de arroz; grama cuiabana e sobras de silagem de milho), e 5 repetições. A avaliação foi realizada 30 dias após a semeadura, quanto aos caracteres de biomassa fresca total, biomassa fresca das folhas, biomassa fresca da raiz tuberosa, produtividade comercial e relação de folhas e raízes. Foram verificadas diferenças significativas $(p<0,05)$ entre as coberturas vegetais utilizadas em todos os caracteres estudados, com exceção da relação de folhas e raízes. Em relação à biomassa fresca da raiz tuberosa e produtividade comercial, a casca de arroz se destacou como cobertura morta vegetal, tendo apresentado os melhores resultados. O bagaço de cana-de-açúcar foi a cobertura morta vegetal que conferiu pior desempenho para os caracteres de biomassa fresca total e a biomassa fresca das folhas. Sendo assim, é possível concluir que a casca de arroz como cobertura morta vegetal proporcionou maior desempenho agronômico à cultura do rabanete, em especial aos caracteres de biomassa fresca total e de produtividade comercial que são comercialmente os mais relevantes.
\end{abstract}

Palavras-chave: Bagaço de cana-de-açúcar. Casca de arroz. Mulching. Resíduos orgânicos. Silagem.

\section{AGRONOMIC PERFORMANCE OF RADISH UNDER DIFFERENT VEGETABLES MULCHES ON SOIL}

\begin{abstract}
SUMMARY: The use of organic waste in agriculture from the most varied areas of the food production chain is extremely important, in view of its polluting potential and as a source of nutrients for the soil. In this sense, the present study aimed to evaluate the influence of organic residues as vegetable mulch on the agronomic performance of radish culture (Raphanus sativus. L.). The experiment was carried out in a randomized block design (RBD), with 4 treatments (sugarcane bagasse; rice hulls; cuiabana grass and corn silage), and 5 replications. Radishes were evaluated at 30 days after sowing for total fresh biomass, fresh leaf biomass, fresh tuber biomass, commercial productivity and the leaf-to-root ratios. Significant differences $(p<0,05)$ were found between the vegetables mulches used in all the studied characters, exception of the leaf-to-root ratio. Regarding the fresh tuber biomass and commercial productivity, the rice hulls stood out as the vegetal mulch that had presented the best results. Sugarcane bagasse was the vegetable mulch that provided the worst performance for the characters of total fresh biomass and fresh leaf biomass. Thus, it is possible to conclude that the rice hulls is the vegetable mulch, which provided higher agronomic performance to the radish culture, especially to the characters of total fresh biomass and commercial productivity, which are the most commercially relevant.
\end{abstract}

Keywords: Sugarcane bagasse. Rice hulls. Mulching. Organic waste. Silage.

\footnotetext{
${ }^{1}$ Universidade Federal de Uberlândia, Campus Glória, Uberlândia, MG, Brasil

Universidade Federal de Uberlândia, Campus Umuarama, Uberlândia, MG, Brasil

${ }^{3}$ Universidade do Estado de Minas Gerais, Unidade Ituiutaba, Ituiutaba, MG, Brasil
}

Nucleus, v.17, n.2, out. 2020 


\section{INTRODUÇÃO}

A cobertura do solo traz diversas vantagens ao sistema produtivo das olerícolas, tais como, a redução da evapotranspiração do solo, controle de plantas infestantes, redução das oscilações de temperatura do solo, proporciona a maior precocidade e rendimento das culturas (TEÓFILO et al., 2012).

Segundo Costa et al. (2013) a cobertura do solo funciona como uma camada atenuadora ou dissipadora de energia, que promove a proteção do solo contra o impacto direto das gotas de chuva, atua como obstáculo ao movimento do excesso de água que não infiltrou no solo e impede o transporte e o arrastamento de partículas pela enxurrada.

Dentre as diversas culturas olerícolas que fazem uso da cobertura do solo está a do rabanete, cujo qual apresenta um ciclo extremamente curto, sendo muito sensível as mudanças ambientais abruptas como, exemplo, as condições físico-hídricas do solo, sendo assim, uma cultura muito sensível à redução dos níveis de água no solo (SILVA et al., 2012).

Nas últimas décadas, tem sido dada atenção cada vez maior para o reaproveitamento de resíduos sólidos que são gerados nas mais diferentes áreas da cadeia produtiva de alimentos, sendo, os principais resíduos, a casca, a palha, o caroço, dentre outros que apresentam características nutricionais importantes, que os tornam passíveis de reuso (GASTL FILHO; LABEGALINI, 2017)

De acordo com Rosa et al. (2011) o descarte de resíduos orgânicos representa a perda de nutrientes e de biomassa e quando descartado de forma inadequada se trata de um potencial poluidor dos solos e dos corpos hídricos que associado à sua lixiviação de compostos pode acarretar problemas de saúde pública.

Na região do Pontal do Triângulo Mineiro existem diversas agroindústrias que geram resíduos orgânicos, tais como Usina BP Biocombustíveis, Usina Santa Vitória, Usina CRV, que produzem bagaço de cana-de-açúcar e a Arroz Dolar que é responsável pela geração de casca de arroz. Além do mais, existem sobras de silagem de milho nas propriedades rurais da região, bem como extensas áreas cultivadas com grama cuiabana que quando podadas podem ser reutilizadas para outras finalidades. Estes resíduos orgânicos poderiam vir a ser empregados como cobertura do solo em diversas culturas agrícolas ou mesmo serem incorporadas ao solo, o que faria com que o teor de matéria orgânica no perfil do solo fosse elevado.

O uso de resíduos orgânicos como cobertura do solo é benéfica, pois promove o aumento da matéria orgânica e é fonte de energia para a microbiota do solo, que, por sua vez, realiza a mineralização dos nutrientes, tornando-os disponíveis para as plantas e influenciando positivamente os índices de produtividade das culturas (COSTA et al., 2013). 
Neste sentido, o presente estudo teve por objetivo avaliar a influência de resíduos orgânicos como cobertura morta vegetal sobre o desempenho agronômico da cultura do rabanete (Raphanus sativus L.).

\section{MATERIAL E MÉTODO}

O estudo foi realizado na Fazenda Experimental de Ituiutaba (FAEXPI) da Universidade do Estado de Minas Gerais (MG), Unidade Ituiutaba, cujo georeferenciamento é $18^{\circ} 57^{\prime} 03^{\prime}$ de latitude S, 4931'31" de longitude W e $560 \mathrm{~m}$ de altitude no município de Ituiutaba (MG). O clima da região é classificado em $A w$ de acordo com a classificação climática de Köppen e Geiger (1928), a temperatura média é de $23,9^{\circ} \mathrm{C}$ e a precipitação média anual é de $1.352 \mathrm{~mm}$. O período do estudo foi de abril a julho de 2017. O solo foi classificado como Latossolo Vermelho eutroférrico de textura média (EMBRAPA, 1999).

O experimento foi conduzido em delineamento em blocos casualizado (DBC), com 4 tratamentos (bagaço de cana-de-açúcar; casca de arroz; grama cuiabana e sobras de silagem de milho), e 5 repetições, totalizando 20 parcelas. As parcelas receberam quantidade da cobertura morta vegetal suficiente para cobrir os canteiros, perfazendo uma espessura em torno de $3,0 \mathrm{~cm}$.

O rabanete cultivar Redondo Vermelho Gigante foi semeado através de semeadura direta em canteiros de dimensão de $1,5 \mathrm{~m}$ x 1,5 m e altura de 20,0 cm, com espaçamento de $15,0 \mathrm{~cm}$ entre linhas e 5,0 cm entre plantas, perfazendo 20 plantas por metro linear. A área útil consistiu das 5 linhas centrais e as 10 plantas centrais de cada linha da unidade experimental.

Com exceção da casca de arroz, o bagaço de cana-de-açúcar, a grama cuiabana e as sobras de silagem, foram triturados em desintegrador, peneiradas e colocadas para secar ao sol, posteriormente foi realizada a sua distribuição uniforme em cada unidade experimental após a semeadura. Foi aplicado no momento do plantio uma dose única de $300,0 \mathrm{~kg} \mathrm{ha}^{-1}$ do adubo 08 28-16, correspondente a 7,0 $\mathrm{g} \mathrm{linha}^{-1}$ da referida fórmula, conforme recomendado para cultura pela quinta aproximação para o Estado de Minas Gerais.

O experimento foi avaliado aos 30 dias após a semeadura, quanto aos caracteres de biomassa fresca total (BFT), biomassa fresca das folhas (BFF), biomassa fresca da raiz tuberosa (BFRT), produtividade comercial (PC) e a relação de folhas e raízes (F/R).

A BFT foi obtida por meio da pesagem da planta inteira de rabanete, em balança eletrônica com precisão de $0,001 \mathrm{~g}$. A BFF, por sua vez, foi obtida por meio da pesagem das folhas de rabanete em balança eletrônica com precisão de $0,001 \mathrm{~g}$. Por fim, a BFRT foi obtida através da pesagem dos tubérculos de rabanetes em balança eletrônica com precisão de $0,001 \mathrm{~g}$. A PC $\left(\mathrm{t} \mathrm{ha}{ }^{-1}\right)$ foi estimada por meio da Equação 1, considerando a densidade de semeadura do presente estudo. 


$$
\text { PC }\left(\mathrm{t} \mathrm{ha}^{-1}\right)=\text { BFRT } \times 1.333 .333 \text { plantas ha }{ }^{-1} \times 0,001(1)
$$

Em que: PC é a produtividade comercial $\left(\mathrm{t} \mathrm{ha}^{-1}\right)$, BFRT é biomassa fresca da raiz tuberosa $\left(\mathrm{g}\right.$ planta $\left.^{-1}\right)$.

Após a coleta dos dados, os valores foram submetidos ao teste de Shapiro-Wilk para verificar a normalidade dos resíduos e ao teste de Bartlett para verificar a homocedasticidade. Posteriormente, realizou-se a análise de variância (ANOVA) e as médias dos tratamentos foram comparadas entre si, pelo teste de comparação múltipla de Tukey a uma probabilidade de 5,0\% de erro. O Programa estatístico utilizado foi o R version 3.6.2 (Dark and Stormy Night) (R CORE TEAM, 2019).

\section{RESULTADO E DISCUSSÃO}

Foram verificadas diferenças significativas $(p<0,05)$ entre as coberturas utilizadas para a cultura do rabanete para todos os caracteres avaliados, em outras palavras, as coberturas de solo utilizada afetaram de maneira significativa todos os caracteres agronômicos estudados (Tabela 1).

Tabela 1. Biomassa fresca total (BFT), biomassa fresca das folhas (BFF), biomassa fresca da raiz tuberosa (BFRT), produtividade comercial (PC) e relação de folhas e raízes (F/R) de rabanete cultivar Redondo Vermelho Gigante em função da cobertura do solo.

\begin{tabular}{|c|c|c|c|c|c|}
\hline \multirow{2}{*}{ Cobertura morta vegetal } & BFT & $\mathrm{BFF}$ & BFRT & $\mathrm{PC}$ & \multirow{2}{*}{$\mathrm{F} / \mathrm{R}$} \\
\hline & \multicolumn{3}{|c|}{------------- g planta ${ }^{-1}$------------- } & ---- $\mathrm{t} \mathrm{ha}^{-1}$---- & \\
\hline Bagaço de cana-de-açúcar & $29,800 \mathrm{~b}$ & $6,800 \mathrm{~b}$ & $23,000 \mathrm{~b}$ & $30,667 \mathrm{~b}$ & $0,2972 \mathrm{a}$ \\
\hline Grama cuiabana & $35,080 \mathrm{a}$ & $9,200 \mathrm{a}$ & $25,880 \mathrm{~b}$ & $34,507 \mathrm{~b}$ & $0,3548 \mathrm{a}$ \\
\hline Sobras de silagem de milho & $35,360 \mathrm{a}$ & $9,600 \mathrm{a}$ & $25,760 \mathrm{~b}$ & $34,347 \mathrm{~b}$ & $0,3728 \mathrm{a}$ \\
\hline Casca de arroz & $38,080 \mathrm{a}$ & $9,120 \mathrm{a}$ & $28,960 \mathrm{a}$ & 38,613 a & $0,3154 \mathrm{a}$ \\
\hline Teste de F & $15,614 *$ & $8,443^{*}$ & $12,579 *$ & $12,579 *$ & $3,520^{\mathrm{ns}}$ \\
\hline $\mathrm{CV}^{1}(\%)$ & 5,67 & 11,27 & 5,93 & 5,93 & 12,38 \\
\hline Normalidade $^{2}$ & $\mathrm{~ns}$ & ns & ns & ns & ns \\
\hline Homogeneidade $^{3}$ & $\mathrm{~ns}$ & ns & ns & ns & ns \\
\hline
\end{tabular}

Médias seguidas por letras distintas nas coluna se diferem pelo teste de Tukey a uma probabilidade de 5,0\% de erro. *Significativo pelo teste de $\mathrm{F}$ a uma probabilidade de 5,0\% de erro. ${ }^{\text {ns }}$ Não significativo. ${ }^{1}$ Coeficiente de variação. ${ }^{2}$ Normalidade dos resíduos pelo teste de Shapiro-Wilk, em nível de 5,0\% de probabilidade de erro. ${ }^{3}$ Homogeneidade das variâncias pelo teste de Oneill e Mathews, em nível de 5,0\% de probabilidade de erro.

Fonte: Dados da pesquisa, 2017. 
As coberturas vegetais de grama cuiabana, sobras de silagem de milho e casca de arroz apresentaram significativamente $(p<0,05)$ os maiores valores de BFT, sendo superior ao bagaço da cana-de-açúcar como cobertura.

Resultados superiores foram obtidos por Almeida et al. (2019) para rabanete cv. Crimson Gigante com valores superiores a 40,0 $\mathrm{g} \mathrm{planta}^{-1}$ em BFT.

Segundo Rodriguez et al. (2017) os caracteres biomassa verde e seca de raiz, bem como de diâmetro de raiz, são comercialmente relevantes, uma vez que o seu peso e tamanho afetam diretamente a qualidade da raiz de rabanete e, consequentemente, no seu valor.

Do mesmo modo que em BFT, as coberturas vegetais de grama cuiabana, sobras de silagem de milho e casca de arroz proporcionaram significativamente $(p<0,05)$ maior ganho de BFF para as plantas de rabanete, enquanto que o bagaço de cana-de-açúcar teve significativamente $(p<0,05)$ o pior desempenho como cobertura vegetal do solo.

Kramer (2018) ao avaliarem a BFF das cultivares de rabanete Gigante Siculo e Crimson Gigante em dois ciclos obtiveram, respectivamente, 7,73 e 11,03 g planta ${ }^{-1}$ e 4,84 e 13,32 g planta $^{-1}$.

Elsenbach et al. (2017) ao avaliarem a BFF em função das doses de fósforo obtiveram para a cultivar Gigante do Século valores de $6,72 \mathrm{~g} \mathrm{planta}^{-1}$ para a testemunha e 28,36 $\mathrm{g}$ planta $^{-1}$ para a maior dose de fósforo aplicada. Resultados superiores foram obtidos por Rocha (2018)

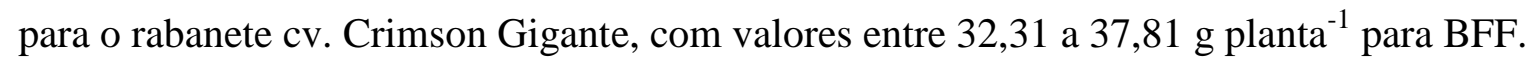

Ao avaliar os efeitos de diferentes doses de cinzas no cultivo de rabanete cv. Crimson Gigante, Gastl Filho et al. (2019) obtiveram valor máximo de 10,29 g planta ${ }^{-1}$ para BFF, resultados similares ao do presente estudo.

Khazaei et al. (2013) afirma que o uso de coberturas no cultivo de plantas gera um microclima propício ao melhor desenvolvimento das plantas, o que resulta na maior a retenção de umidade e regulação da temperatura do solo, além de suprimir o crescimento de ervas daninhas na área de cultivo.

Quando observada a BFRT, a casca de arroz foi a cobertura vegetal que proporcionou significativamente $(p<0,05)$ o maior acúmulo de biomassa fresca para os tubérculos de rabanete, enquanto que os menores valores significativamente $(p<0,05)$ menores foram observados para as coberturas vegetais de grama cuiabana, bagaço de cana-de-açúcar e sobras de silagem de milho.

Resultado similar ao do presente estudo foi encontrado por Tanaka e Queiroz (2017) que ao avaliarem a casca de arroz como cobertura morta vegetal para a cultura do rabanete, obtiveram BFRT de 37,0 g planta ${ }^{-1}$. Gastl Filho et al. (2019) obtiveram para o rabanete cv. Crimson Gigante valor máximo de 22,16 g planta $^{-1}$ para BFRT, resultados similares ao do presente estudo. 
Em seu estudo Bulegon et al. (2012) obtiveram valores entre 20,277 e 25,043 g planta $^{-1}$ para BFRT. Ao estudar diferentes dosagens de fósforo na cultura do rabanete Elsenbach et al. (2017) obtiveram para a cultivar Apolo valores de BFRT entre 25,31 a 29,31 g planta $^{-1}$.

Valores inferiores foram verificados por Galvão (2008) para BFRT, utilizando semeadura direta em cobertura morta e preparo de solo convencional (sem cobertura), com valores médios de 13,78 e 14,09 g planta $^{-1}$, respectivamente. Da mesma forma, Bonela et al. (2017), obtiveram para as cultivares de rabanete Vip Crimson e RedJewel F1, valores médios de 2,92 e 5,18 g planta $^{-1}$, respectivamente.

A BFRT obtida por Kramer (2018) para as cultivares Gigante Siculo e Crimson Gigante cultivados em dois ciclos foram, respectivamente, 14,90 e 10,73 $\mathrm{g} \mathrm{planta}^{-1}$ e 11,49 e 12,60 g planta $^{-1}$, sendo inferior ao do presente estudo. Resultados superiores foram obtidos por Rocha (2018) para o rabanete cv. Crimson Gigante, com valores entre 29,75 a 40,37 $\mathrm{g}$ planta $^{-1}$ para BFRT.

Rodrigues et al. (2009) afirmaram que a adição de cobertura morta ao solo em níveis adequados passa por degradação promovida pela biomassa microbiana presente no solo, levando à mineralização dos nutrientes, podendo ter efeito imediato ou residual.

Do mesmo modo que para BFRT, a PC estimada da cultura do rabanete utilizando a casca de arroz como cobertura morta do solo se mostrou significativamente $(p<0,05)$ superior às demais utilizadas, com valor de $38,613 \mathrm{t} \mathrm{ha}^{-1}$, enquanto que as demais coberturas vegetais proporcionaram produtividade inferiores a $34,507 \mathrm{t} \mathrm{ha}^{-1}$.

Valores inferiores foram encontrado por Batista et al. (2013) ao avaliarem a PC de tubérculos de rabanete, obtiveram valores entre 3,10 a 12,04 $\mathrm{t} \mathrm{ha}^{-1}$. Do mesmo modo, Rocha (2018) obteve valores inferiores ao do presente estudo para o rabanete cv. Crimson Gigante, com valores entre 7,40 a 8,89 $\mathrm{t} \mathrm{ha}^{-1}$ para produtividade comercial. Em seu estudo, Bulegon et al. (2012) verificaram para PC de rabanete cultivado em 7,0 t ha ${ }^{-1}\left(3,463\right.$ a 6,120 tha $\left.\mathrm{th}^{-1}\right)$ e 11,0 t ha (2,043 a 3,686 $\left.\mathrm{t} \mathrm{ha}^{-1}\right)$ de palhada de feijão de porco, sendo estes valores inferiores ao solo descoberto $\left(5,089\right.$ a 8,261 $\left.\mathrm{t} \mathrm{ha}^{-1}\right)$.

Não foram verificadas diferenças significativas $(p<0,05)$ entre as coberturas morta vegetal em relação à $\mathrm{F} / \mathrm{R}$, ou seja, as coberturas não foram responsáveis por incrementar ou mesmo reduzir a proporção existente entra a parte aérea do rabanete e a parte radicular do mesmo.

Em seu estudo, Kosterna (2014) verificou que os diferentes tipos de cobertura do solo influenciaram significativamente os aspectos produtivos da cultura do brócolis, tendo aumentado o rendimento, o peso da cabeça comercial e melhorado a qualidade da cabeça, comparado ao obtido no cultivo sem cobertura vegetal. 
As massas verde e seca de raiz, assim como o diâmetro de raiz são parâmetros que comercialmente interessam, pois, o tamanho e o peso interferem na qualidade da raiz de rabanete e, consequentemente, no seu valor (RODRIGUEZ et al., 2017).

Santos et al. (2017) obtiveram resultados distintos ao presente estudo com a utilização da casca de arroz como cobertura morta vegetal na cultura do alface, em que verificaram que a cobertura do solo não proporcionou incrementos de produtividade. Do mesmo modo Fortes Neto et al. (2017) não constataram incrementos de produtividade no cultivo de pimentão ao se utilizar casca de arroz como cobertura morta vegetal.

Por sua vez, Godoy (2016) verificou que o uso de resíduos de grama e da casca de arroz como cobertura morta vegetal, incrementou a produtividade quando comparada a testemunha sem nenhuma cobertura e a outros tipos de cobertura estudados.

Kramer (2018) obteve para a cobertura morta vegetal de Braquiária no primeiro e segundo ciclos valores de 6,41 e 13,15 g planta ${ }^{-1}$ para BFF e 13,76 e 13,15 g planta $^{-1}$ para BFRT, respectivamente, sendo valores similares para a BFF e inferiores para BFRT do rabanete cultivado utilizando como cobertura morta grama cuiabana.

\section{CONCLUSÃO}

É possível concluir que a casca de arroz como cobertura morta vegetal proporcionou maior desempenho agronômico à cultura do rabanete, em especial às características de BFRT e produtividade comercial que são comercialmente os mais relevantes.

\section{REFERÊNCIAS}

ALMEIDA, R. H. B. DE; SILVA, I. H. DE O.; MONTEIRO, D. N. R.; ROSA, N. L.; TONELLO, A. P. Níveis de água no solo na produção de rabanete utilizando tensiômetro. In: ENCONTRO INTERNACIONAL DE PRODUÇÃO CIENTÍFICA, 11., 2019, Maringá. Anais [...]. Maringá: UniCesumar, 2019. p. 1-4.

BATISTA, M. A. V.; BEZERRA NETO, F.; AMBRÓSIO, M. M. Q.; GUIMARÃES, L. M. S.; SARAIVA, J. P. B.; SILVA, M. L. da. Atributos microbiológicos do solo e produtividade de rabanete influenciados pelo uso de espécies espontâneas. Horticultura Brasileira, Vitoria da Conquista, v. 31, n. 4, p.587-594, Dez. 2013. https://doi.org/10.1590/S0102$\underline{05362013000400013 .}$.

BONELA, G. D.; SANTOS, W. P. dos; SOBRINHO E. A.; COSTA GOMES E. J. da. Produtividade e qualidade de raízes de rabanete cultivadas sob diferentes fontes residuais de matéria orgânica. Revista Brasileira de Agropecuária Sustentável, v.7, n.2, p. 66-74, 2017

BULEGON, L. G.; FERREIRA, S. D.; YASSUE, R. M.; MOREIRA, G. C.; PASTÓRIO, M. A.; FARIAS, F. B. Desenvolvimento e produtividade de rabanete sobre diferentes deposições de palhada de feijão de porco e doses de nitrogênio. Cultivando o Saber, Cascavel, v.5, n.4, p. 191202, 2012. 
COSTA, E. M.; SILVA, H. F.; RIBEIRO, P. R. A. Matéria orgânica do solo e o seu papel na manutenção e produtividade dos sistemas agrícolas. ENCICLOPÉDIA BIOSFERA, Centro Científico Conhecer - Goiânia, v.9, n.17; p.1842-1860, 2013.

ELSENBACH, H.; FONTINELLI, A. M.; MARENGO, R, P.; RIBEIRO, G. Doses de fósforo submetidas a diferentes cultivares de rabanete (Raphanus sativus L.). In: SALÃO INTERNACIONAL DE ENSINO, PESQUISA E EXTENSÃO, 9., 2017, Fronteira da Paz. Anais [...]. Fronteira da Paz: UFP. 2019. p. 1-6.

EMBRAPA - EMPRESA BRASILEIRA DE PESQUISA AGROPECUÁRIA. Sistema brasileiro de classificação de solos. Brasília - DF, 1999. 412p.

FORTES NETO, P.; DUARTE, E.; FORTES, N.; SARAIVA, A.; COSTA, R.; SILVA, E. Efeitos de diferentes tipos de cobertura de solo no cultivo de pimentão. In: CONGRESSO LUSOBRASILEIRO DE HORTICULTURA, 1., 2017, Lisboa. Anais [...] . Lisboa: Aph, 2017. p. 456463.

GALVÃO, R. O. Produção orgânica de hortaliças em diferentes sistemas de plantio com cobertura viva e morta adubado com composto, no estado do Acre. 2008. 64p. Dissertação. (Mestrado em Produção Vegetal) - Universidade Federal do Acre, Rio Branco - AC, 2008.

GASTL FILHO, J.; ARAUJO, R. da S.; FERREIRA, I.; MARTINS, I. S.; PIVA, H. T.; RESENDE, M. A. Potencial corretivo e multinutriente de cinzas de biomassa vegetal na cultura do rabanete sob manejo orgânico. In: CICLO DE SEMINÁRIOS DE AGRONOMIA, 12., 2019, Uberlândia, Anais [...]. Uberlândia: PET Agronomia UFU, 2019, v. 12, p. 421-425.

GASTL FILHO, J.; LABEGALINI, M. C. Desenvolvimento de licor à base de cascas de abacaxi. In: SEMINÁRIO DE PESQUISA E INOVAÇÃO TECNOLÓGICA. 1., 2017, Uberaba, Anais [...]. Uberaba: Instituto Federal do Triângulo Mineiro, 2017, v.1, p.1-8.

GODOY, R. B. Efeitos de cobertura no solo com resíduos vegetais na qualidade pós-colheita de cenoura. 2016. 17 f. TCC (Graduação) - Curso de Agronomia, Instituto Federal de Educação Ciência e Tecnologia Goiano, Morrinhos, 2016.

KHAZAEI, I.; REZA, S.; ABDOLKARIM, K.; MOHAMMAD, S. M.; MIRJALILI, S. M. Improvement of lettuce growth and yield with spacing, mulching and organic fertilizer. International Journal of Agriculture and Crop Sciences, v. 6, p. 1137-1143, 2013.

KÖPPEN, W.; GEIGER, R. Klimate der Erde. Gotha: Verlag Justus Perthes. 1928.

KOSTERNA, E. Soil mulching with straw in broccoli cultivation for early harvest. Journal of Ecological Engineering, v. 15, p. 100-107, 2014.

KRAMER, M. Produção de cultivares de rabanete em função de plantas de cobertura em antecedência à semeadura. 2018. 47 f. Dissertação (Mestrado) - Curso de Mestrado Profissional em Olericultura, Instituto Federal de Educação, Ciência e Tecnologia Goiano, Morrinhos, 2018.

R CORE TEAM. R: A language and environment for statistical computing. R Foundation for Statistical Computing, Vienna, Austria. 2019. 
ROCHA, T. A. L. C. G. Efeito da adubação orgânica com diferentes compostos orgânicos na cultura do rabanete, no semiárido brasileiro. 2018. 18 f. Dissertação (Mestrado) - Curso de Mestrado Profissional em Uso Sustentável de Recursos Naturais, Instituto Federal de Educação, Ciência e Tecnologia do Rio Grande do Norte, Natal, 2018.

RODRIGUES, D. S.; NOMURA, E. S.; GARCIA, V. A. Coberturas de solo afetando a produção de alface em sistema orgânico. Revista Ceres, v.56, n.3, p. 332-335, 2009.

RODRIGUEZ, D.P.; TONIETTO, S.M.; PIESANTI, S.R.; HENRIQUEZ, J.M.; SCHUBERT, R.; MORSELLI, T.B.G. A. Produção de rabanete (Raphanus sativus 1.) sob o residual do cultivo orgânico da alface. Revista da Jornada de Pós-Graduação e Pesquisa, Urcamp, v.14, n.14, p.2329-2340, 2017.

ROSA, M. F.; SOUZA FILHO, M S. M.; FIGUEIREDO, M. C. B.; MORAIS, J. P. S.; SANTAELLA, S.T.; LEITÃO, R.C. Valorização de resíduos da agroindústria. In: SIMPÓSIO INTERNACIONAL SOBRE GERENCIAMENTO DE RESÍDUOS AGROPECUÁRIOS E AGROINDUSTRIAIS, 2., 2011, Foz do Iguaçu. Anais [...], Foz do Iguaçu: Sociedade Brasileira dos Especialistas em Resíduos das Produções Agropecuária e Agroindustrial, 2011. v. 1, p. 98 105.

SANTOS, J. R. C. DOS; FERNANDES, C. N. V.; OLIVEIRA FILHO, J. N.; SILVA, A. R. A. DA; FERNANDES, J. N. V.; SARAIVA, K. R. Adubação nitrogenada e cobertura do solo no cultivo da alface irrigada. Revista Brasileira de Agricultura Irrigada, v.12, n.1, p.2327-2337, 2018.

SILVA, R. T.; SOUZA, A. A. T.; OLIVEIRA, F. A.; TARGINO, I. S. O.; SILVA, M. L. do N. Tolerância do rabanete ao encharcamento do solo. Revista Verde, Mossoró, RN, v. 7, n.1, p. 2533, jan./mar. 2012.

TANAKA, D. V.; QUEIROZ, F. G. Influência de diferentes tipos de cobertura de solo na cultura do rabanete. e-RAC, v. 7, n. 1, 2018.

TEÓFILO, T. M. S.; FREITAS, F. C. L.; MEDEIROS, J. F.; FERNANDES, D.; GRANGEIRO, L. C.; TOMAZ, H. V. Q.; RODRIGUES, A. P. M. S. Eficiência no uso da água e interferência de plantas daninhas no meloeiro cultivado nos sistemas de plantio direto e convencional. Planta Daninha, Viçosa, v. 30, n. 3, p.547-556, 2012. 